\title{
VSD 引流围手术期护理对于治疗效果的影响的临床研究
}

\section{Clinical Study on the Effect of Perioperative Nursing on the Treatment Effect of VSD Drainage}

\author{
曲瑞红 \\ Ruihong Qu \\ 呼和浩特市蒙医中医医院 中国·内蒙古 呼和浩特 010010
}

Hohhot Mongolian Medicine and Traditional Chinese Medicine Hospital, Hohhot, Inner Mongolia, 010010, China

\begin{abstract}
摘 要: 目的: 探究 VSD 引流围手术期护理对于治疗效果的影响的临床研究。方法:选取 2019 年 5 月至 2019 年 8 月呼和浩 特市蒙医中医医院西医骨科收治的 80 例开放性骨折需要 VSD 装置手术的患者, 随机分为实验组、对照组, 每组均为 40 例患 者。对照组患者行常规骨科护理, 实验组患者在传统护理的基础上围手术期护理宣教;对比两组患者的 VSD 出现故障的概 率。结果: 根据实验结束后 7 天内 VSD 的使用情况可知, 实验组出现故障共有 3 例, 其中漏气 2 例, 漏液 1 例, 由于发现及时, 都完善处置; 对照组出现故障 13 例, 其中漏气 5 例, 漏液 4 例, 已完善处理,鼓起 2 例, 其中 1 例因发现较晚, VSD 未能达到预 期效果。对数据进行统计学分析, 进行 $\chi^{2}$ 检验, $P<0.05$, 差距具有统计学意义。结论: VSD 引流围手术期护理能够显著降低故 障率, 提高患者的满意度。
\end{abstract}

\begin{abstract}
Objective: To explore the clinical effect of perioperative nursing on the treatment effect of VSD drainage. Methods: To select 80 patients with open fracture who need VSD device in Hohhot Mongolian Medicine and Traditional Chinese Medicine Hospital from May 2019 to August 2019, and randomly divide them into experimental group and control group with 40 patients in each group. The patients in the observation group received perioperative nursing education on the basis of traditional nursing. The probability of VSD failure in the two groups was compared. Results: The use of VSD within 7 days after the end of the experiment, it can be seen that there were 3 cases of failure in experimental group, including 2 cases of air leakage and 1 case of liquid leakage. Due to the timely discovery, the treatment was improved. In control group, there were 13 cases of failure in the control group, including 5 cases due to air leakage, 4 cases due to liquid leakage, 2 cases due to complete treatment, and 1 case due to late detection, VSD failed to achieve the expected effect. By statistical analysis of data, $\chi^{2}$ test was performed, $P<0.05$, the gap is statistically significant. Conclusion: VSD drainage perioperative nursing can significantly reduce the failure rate and improve patients' satisfaction.
\end{abstract}

关键词: VSD; 围手术期护理;治疗效果

Keywords : VSD; perioperative nursing; treatment effect

DOI : $10.36012 /$ pmr.v2i3.2287

\section{1 引言}

随着科学技术的发展, 越来越多的技术、材料应用于医 疗工作中,负压封闭引流技术 (Vacuum Sealing Drainage,VSD) 就是其中之一。VSD 是用内含有硅胶多孔引流管的医用海绵 敷料来覆盖或填充皮肤、软组织缺损的创面, 再用单向透气 的生物半透膜对之进行封闭, 使其成为一个密闭空间, 最后 把引流管接通负压源,通过可控制的负压来促进创面愈合的
一种全新的治疗方法。VSD 目前广泛用于皮肤损伤、肌肉骨 骼外露无法完成一期缝合、糖尿病足、皮肤感染等领域, 并取 得了良好的疗效,但前提是 VSD 装置能够稳定良好地运行[1]。 由于 VSD 装置需要在患者体外安装一段时间，在这一段时 间内降低故障率，保证治疗效果是临床工作的重中之重,而 针对围手术期最主要的护理人员的护理更是关键, 本研究通 过对比传统护理与 VSD 围手术期护理，对患者的治疗效果 
进行临床研究。

\section{2 资料与方法}

\section{1 一般资料}

选取 2019 年 5 月至 2019 年 8 月呼和浩特市蒙医中医 医院西医骨科收治的 80 例开放性骨折需要 VSD 装置手术 的患者, 随机分为实验组和对照组, 每组各 40 例患者。其中, 实验组男性 18 例, 女性 22 例; 对照组男性 21 例, 女性 19 例。选择的患者年龄相仿, 并且病情严重程度大体一致, 以求 在年龄和性别等方面比较差异无统计学意义 $(P>0.05)$ 。

\section{2 方法}

实验组和对照组分别行 VSD 负压吸引术, 麻醉成功后, 彻底清创, 完成骨折相关复位, 外固定后, 在受伤创面覆盖 VSD 敷料, 摆放好引流管, 薄膜密封, 检查气密性。持续负压 吸引 1 周。

实验组: 对于实验组患者, 围手术期护理宣教及精细护 理。护理人员术前告知患者此类骨折需行 VSD 术, 并详细讲 解 VSD 的概念、用法及使用时限。护理人员于术前协助医生 对患者进行初步的皮肤消毒、清创、包扎。由于此类患者多为 剧烈疼痛, 护理人员需耐心交流, 多讲解 VSD 成功典型并展 示相应的图片或视频,消除患者的恐惧心理。术后的护理是 VSD 的重中之重, 交接好负压吸引装置, 因为一次性 VSD 装 置要持续吸引 1 周, 放置位置既要有利于引流液体的排出, 还要便于患者活动。VSD 术后出现皮肤边缘与贴膜粘贴不牢 靠, 出现漏气漏液是常见的问题, 定期检查装置的气密性, 定 期冲管, 检查有无管道因坏死物质堵塞, 如有上述现象应及 时向医生报告。调节负压装置, 维持在 $500 \mathrm{mmHg}$ 左右, 压力 不足直接影响引流效果，压力过大临床上多见吸引管变㾴， 无法流通, 护理人员密切观察的同时告知患者观察重点。每 日记录引流液体量、颜色, 观察 VSD 周围的皮肤感觉及血运 情况。临床常出现 VSD 安装后创面周围过敏、出血、出现水 泡,没有第一时间发现而失败的案例, 对患者的精神和经济 造成损失, 因此护理观察尤为重要。一般来说, 早期及时的装 置调整对于治疗效果影响很小。同时, 应及时地指导患者正 确进行功能锻炼, 保障关节功能的同时避免 VSD 装置脱落。

对照组: 完善常规入院宣教, 未有 VSD 相关材料, 装置 放置于常规放置位置, 未告知患者相关需要观察 VSD 的气
密性重点。执行常规骨折病人的医师医嘱及功能锻炼。

\section{3 观察指标}

护理过程中根据患者出现的漏气、漏液、吸引失效、装置 鼓起等情况,计算两组患者的故障率。

\section{4 统计学处理}

对两组数据进行统计学分析, 运用 SPSS21.0, 计量资料 采用 $\chi^{2}$ 检验, 分析差异性, 计算 $P$ 值, $P<0.05$ 说明两组数据 之间具有显著差异性。

\section{3 结果}

根据 7 天内 VSD 的使用情况可知，实验组出现故障共 有 3 例, 其中漏气 2 例, 漏液 1 例, 由于发现及时, 都完善处 置。对照组出现故障 13 例, 其中漏气 5 例, 漏液 4 例, 已完善 处理,鼓起 2 例, 其中 1 例因发现较晚, VSD 未能达到预期效 果。对数据进行统计学分析, 进行 $\chi^{2}$ 检验, $P<0.05$, 说明差距 具有统计学意义。实验组与对照组的故障比较如表 1 所示。

表 1 实验组与对照组的故障比较

\begin{tabular}{c|c|c|c}
\hline 组别 & 出现故障/例 & 未出现故障/例 & 故障率/\% \\
\hline 实验组 & 3 & 37 & $7 \%$ \\
\hline 对照组 & 11 & 29 & $22 \%$ \\
\hline
\end{tabular}

\section{4 结论}

针对临床中需要使用符合适应证的患者, VSD 是一种效 果较好、方便实用的装置, 它能够减少患者的换药痛苦, 减少 住院时间,减少感染概率, 增强患者的信心, 为二期手术做充 分准备。完善的围手术期护理在患者康复的过程中扮演着十 分重要的角色, 精细的护理工作能够帮助患者更好地理解 VSD 的治疗原理、注意事项、治疗预后, 为患者打消疑虑, 从 而提高患者的配合积极性, 形成良好的医一护一患关系, 为 患者的进一步治疗提供基础。本实验结果也充分说明了积极 的围手术期护理在治疗工作中的重要性,能够提高治疗的成 功率以及患者的满意率 ${ }^{[2]}$ 。

\section{5 结语}

随着 VSD 使用越来越广泛, VSD 的问题也逐渐凸显。其 中, 较为突出的就是 VSD 使用过程中, 由于患者对 VSD 的陌 生及不信任产生的一些问题,漏气、漏液、鼓起等不能及时发 现 ${ }^{[3]}$ 。大部分临床出现失败的主要原因是发现较晚, 患者对

(下转第 43 页) 
Marder VJ,eds.Hemostasis and Thrombosis.Basic principles \&clinical practice [J].4th ed.Philadelphia: Lippincott Williams \&Wilkins,2001(1):1131-1152.

[4] Olsson E,Svartling N,Asko-Seljavaara S,etal.Activation of coagulation and fibrinolysis during reconstructive microsurgery in patients with cancer [J].Microsurgery, 2001(21):208-213.

[5] Kono T,Ohtsuki T,Hosomi N,etal.Clinical characteris tics of ischemic stroke in elderly patients with cancer[J]. Nihon Ronen Igakkai Zasshi,2011,48(1):57-62.

[6] Cushman M,Folsom R,Wang L,etal.Fibrin fragment D dimer and the risk of future venous thrombosis[J].Blood, 2003,101(4):1243-1248.

[7] Riedl J,Pabinger I,Ay C.Platelets in cancer and thrombosis[J]. Hamostaseologie, 2014,34(1):54-62.

[8] John A. Cancer and venous thromboembolism :scope of the problem[J]Cancer Control,2005,12(1):5-10.

[9] Khoury JD,Adcock DM,Chan F, etal.Increases in quantitative D dimer levels correlate with progressive disease better than circulating tumor cell counts in patients with refractory prostate cancer $[\mathrm{J}]$.Am J Clin Pathol,2010,134(6):964-969.

[10] Deme D,Ragán M,Kalmár K,etal.Metastatic prostate cancer complicated with chronic disseminated intravascular coagulopathy causing acute renal failure, mimicking thrombotic thrombocytopenic purpura and hemolytic uremic syndrome:pathomechanism, differential diagnosis and therapy related to a case [J].Magyar Onkologia,2010,54(4):351-357.

[11] Bergamini S,Bellei E, Bonetti LR,et al.Inflammation: animportant parameter in the search of prostate cancer biomarkers[J]. Proteome Sci,2014,12(1):1-12.

[12] 王素侠,范志伟.前列腺癌疾病进展中 D.二聚体检测的意义 $[\mathrm{J}]$. 检验医学与临床,2016,13(24):3541-3543.

[13] 陈涛,邱建宏, 刘健.血清 GPc-3 联合 PSA 检测在前列腺癌诊 断中的临床意义[J].临床误诊误治,2016,29(9):105-107.

\section{（上接第 39 页）}

VSD 的认识不足, 因此护理人员在此过程中尤为重要。作为 第一线的工作人员，护理人员与患者面对面的时间较长, 对 患者的观察较医生有着优势, 对于患者的宣教与交流, 在术 后的及时发现与反馈, 对于临床上患者的治疗及康复不可或 缺 ${ }^{[4]}$ 。本次实验也存在着许多不足, 如由于患者在教育水平上 的差异, 有些患者在护士进行积极宣教后仍对宣教内容存在 不解, 以致出现 VSD 装置故障, 影响实验的客观性与准确 性, 因此面对不同教育水平的患者, 护理人员应采用因人而 异的沟通方式，以确保临床工作落到实处而不是敷衍了事。 可喜的是,近些年随着医疗水平的不断提高, 护理人员的医 疗技能越来越全面, 与医师的临床工作交集逐渐增多, 其与 医师工作的配合越来越默契, 减轻了医师的压力, 同时有利 于患者的康复, 这无疑在促进医疗发展及加强医护合作的道
路上更进了一步, 相信在不久的将来, 护理工作会扮演更为 重要的角色,在病患的诊治及康复过程中为患者提供更好的 服务 ${ }^{[4]}$ 。

\section{参考文献}

[1] 韦艳艳,罗毅,龙春萍,等.自创简易 VSD 联合早期程序化综合治 疗在中华眼镜蛇咬伤中的应用 [J].当代护士(中旬刊),2020,27 (3):71-73.

[2] 刘慧,冀永峰.VSD 治疗创面的护理研究[J].心理月刊,2020,15 (1): 126 .

[3] 朱天存.游离植皮术后 VSD 的应用及护理对策分析[J].名医, 2020(1):224.

[4] 杨琴,陈扶荣.浅论 VSD 治疗技术的术前术后护理要点[J].新疆 中医药,2019,37(6):74-75. 